\title{
La castration à travers les âges
}

\author{
Georges Androutsos* et Spyros Marketos** \\ * Institut d'Histoire de la Médecine, Faculté de Médecine de l'Université d'Athènes \\ ** Fondation Internationale Hippocratique de Cos, Grèce.
}

\section{RESUME}

Les premières castrations furent incontestablement celles d'Osiris par son frère Seth et d'Uranus par son fils Cronos. Les Egyptiens pratiquaient la castration comme punitions des adultères. Les Hébreux l'abominaient, ainsi que les premiers chrétiens. En Russie, depuis le 18e siècle les "Skoptzys" pratiquaient cette intervention abolie par le tsar vers la fin du $19 \mathrm{e}$ siècle. A l'aube du XXème siècle la castration est encore pratiquée chez les Orientaux pour fournir des eunuques aux harems. Pendant plusieurs siècles, les grandes vedettes de l'opéra, ainsi que du chant ecclésiastique, furent des chanteurs castrés.

Mots clés: castration, opéra, église, harem.

\section{LA CASTRATION DANS LA MYTHOLOGIE}

La première castration, à but non curatif, dans l'histoire fut incontestablement celle d'Osiris par son frère cadet Seth qui pour réduire Osiris à néant s'empara du cadavre inerte de son frère et le dépeça en quatorze morceaux qu'il dispersa dans les méandres du delta du Nil. Isis, la fidèle femme d'Osiris, sa magie aidant, parvint à en retrouver treize qui permirent la résurrection spirituelle du dieu. Mais le quatorzième membre ne lui fut pas rendu, empêchant ainsi qu'il pût à nouveau s'incarner. Il s'agissait de son phallus, avec les testicules, qui fut avalé par un poisson, l'oxyrhinque
(10). Parmi les dieux Grecs, Cronos mutila son père Uranus et jeta le trophée à la mer. Certains cultes païens adoptèrent la castration. En particulier, le culte de Cybèle exigeait de ses prêtres l'autoéviration. Cybèle, s'était follement éprise d'un bel adolescent, le berger Attis, passion qu'il partageait avec une égale ardeur. Mais Attis vivait sous la juridiction du roi de Pessinonte qui avait décidé d'en faire son gendre. Plutôt que de trahir sa divine mâ̂tresse, le beau berger préféra commettre l'irréparable: il s'émascula et mourut de sa blessure. Certes, Cybèle intervient aussitôt après et ressuscita son amant. Attis devint ainsi le symbole de la renaissance et de l'immortalité. Mais le culte phrygien se trouvait définitivement entaché d'une pratique barbare et sanglante: l'émasculation volontaire des prêtres de Cybèle, les galles. Cette pratique fut tenace puisque des siècles plus tard l'empereur romain Héliogabale se l'infligeait lui-même.

A Hiérapolis, en Syrie, on célébrait la déesse Astarté. Les prêtres eunuques voués à ce culte ressemblaient tellement à ceux de Cybèle que les Anciens avaient tendance à les confondre. Selon Lucien de Samosate qui assista aux cérémonies d'Hiérapolis: "Lors des cérémonies se font les nouveaux galles. Pendant que le reste joue de la flûte et célèbre les orgies, quelques-uns entrent en fureur et bon nombre, qui n'étaient venus que pour voir, se laissent aller à ce que je vais dire. Le jeune homme décidé à faire ce sacrifice jette à bas ses vêtements, s'avance au milieu de l'assemblée en 
jetant de grands cris, saisit un coutelas et se châtre lui-même, et court par toute la ville tenant en main ce qu'il a coupé. La maison, quelle qu'elle soit, où il jette ce qu'il tenait, lui fournit des habits et des ornements de femme...". Certains galles regrettaient par la suite l'élan hystérique qui les avait conduits à se mutiler,: "Moi, devenir une ménade, moi, un homme stérile? A présent, je déplore ce que j'ai fait, à présent je le regrette...". Mais beaucoup de galles s'accommodaient de leur nouvel état et en profitaient pour se livrer aux pires débauches. Travestis en femme, ils se comportaient en homosexuels passifs et n'hésitaient pas à se livrer à la prostitution [8].

\section{LA CASTRATION DANS L'ANTIQUITE}

La Bible et différents écrits de l'Antiquité prouvent les origines très anciennes de cet acte accompli probablement par des tribus ou des peuples victorieux contre leurs ennemis capturés, afin d'éviter leur reproduction. Ce châtiment semble avoir fort bien traversé les siècles puisqu'il est encore pratiqué de nos jours dans certaines éthnies éthiopiennes. Les Perses pourraient être les plus anciens à avoir utilisé l'éviration, mais il demeure assez difficile d'établir une chronologie parmi toutes les civilisations (indienne, chinoise, arabe...) qui y ont eu recours [5].

Dans le code de Hammurabi (2000 av. J.-C) sont mentionnés des eunuques ainsi que la castration qui était appliquée comme punition pour les crimes sexuels. Selon certains historiens, il existait des eunuques dans la civilisation minoienne en Crète. En Chine la castration se retrouve dans la littérature depuis 1112 av.J.-C. On sait par ailleurs que les Chinois en firent un commerce afin de satisfaire le goût fort répandu dans leur pays pour les jeunes garçons à l'aspect féminin. En Inde, dans les Vedas il est fait mention des eunuques, et sont décrites plusieurs formes de castration chez l'homme et chez l'animal. Du monde indien, et plus particulièrement du mot sanscrit "sastram" (couteau), semblent dériver les termes "castrat" ou "castration". Chez les Grecs la castration était exceptionnelle et chez les Romains elle était pratiquement inexistante, bien qu'elle représentât une forme de punition. Les civilisations grecques et romaines pratiquèrent très largement le commerce des eunuques en provenance d'Afrique ou d'Asie ; on s'était aperçu que les animaux châtrés se montraient en général plus dociles, plus faciles à domestiquer; de là à utiliser des esclaves mutilés, plus "maniables" dans les tâches rebutantes, il n'y avait qu'un pas à franchir. En Egypte la castration à but religieux était défendue sans doute à cause de la légende d'Osiris, mais depuis $1200 \mathrm{avJ}$.-C, on la pratiquait comme punition des adultères. Les Hébreux abominaient la castration et l'accès des eunuques à la prêtrise ou à la congrégation était interdite. La trace la plus ancienne qu'on puisse relever dans la Bible est celle du Deutéronome (XXIII,1), qui rappelle: "L'homme aux testicules écrasés ou à la verge coupée ne sera pas admis à l'assemblée de Yahvé".

\section{LA CASTRATION DANS L'ERE CHRETIENNE}

Dès le début, l'Eglise condamna sévèrement un tel geste, non seulement chez les païens mais chez tous ceux qui, par excès de zèle, interprétaient à la lettre les paroles de l'évangéliste Matthieu (XIX,12 : "Il y a en effet des eunuques qui sont nés ainsi du sein de leur mère, il y a des eunuques qui le sont devenus par l'action des hommes, et il y a des eunuques qui se sont eux-mêmes rendus tels à cause du Royaume des Cieux". Il va de soi que le célibat et la chasteté demandés par Matthieu n'avaient rien à faire d'une authentique castration, ce qui explique que, dès les premiers siècles, l'Eglise fustigea sans équivoque des personnages tels qu'Origène d'Alexandrie qui, en 202, à l'âge de 18 ans s'automutila. La décision du Concile de Nicée, en 325, selon laquelle l'accès à la prêtrise était formellement interdit à tous ceux qui s'étaient castrés volon- 
tairement, n'empêcha pas la présence d'eunuques dans l'armée et l'administration de l'Empire de Byzance. Le cas de l'eunuque Narsès,devenu général de l'Empire de Byzance, est demeuré célèbre. Beaucoup plus connue était la généralisation de la castration dans le monde arabe, par l'intermédiaire des eunuques de harem, à qui revenait la responsabilité de la chasteté des femmes du Sultan. Ce rôle, à priori considéré comme humiliant, n'empêchait pas un grand nombre d'entre eux d'accéder à de très hautes charges, et l'on sait que la Sublime Porte en faisait une consommation énorme, en particulier au sérail de Constantinople [3].

\section{LA CASTRATION ET LES GRANDS CHANTEURS EUNUQUES}

Peu avant la prise de-Constantinople, l'Empire Byzantin avait été le premier à utiliser de façon notoire les eunuques chanteurs dans les églises, comme nous le raconte le canoniste Théodore Balsamon dans son "Commentaire du Nomocanon" au XIIe siècle. Rien d'étonnant à cela lorsqu' on sait l'influence orientale profonde qui marquait les cérémonies byzantines, ainsi que l'importance considérable qui revêtaient la musique et le chant. Un autre cas connu, est celui du castrat grec Manuel qui était parti pour la Russie et avait fondé une école de chant à Smolensk.

Au Moyen Age toute l'Europe fut concernée par le phénomène de la castration qui, toujours utilisée en divers endroits pour torturer les captifs, servait également de châtiment contre ceux qui avaient commis crime ou viol; de plus la Faculté ne se privait pas de l'employer comme thérapie curative ou préventive contre certains maux tels que la lèpre, la folie, l'épilepsie, l'hydrocèle, la goutte et certaines maladies inflammatoires. Longtemps encore on prétendit guérir la hernie par ce moyen: les barbiers ambulants faisaient la castration simultanément à la cure de hernie, ou d'hydro- cèle, et ils avaient constaté depuis longtemps que les enfants qu'ils opéraient d'une hernie congénitale bilatérale et auxquels ils enlevaient par la même occasion les deux testicules se développaient différement des autres en conservant indéfiniment une superbe voix de soprano. On se mit alors à rechercher des enfants qui avaient une belle voix et à les castrer. La castration était pratiquée un peu partout, tant officiellement dans les hôpitaux de certaines grandes villes, pour des raisons médicales, que clandestinement dans les officines de campagne. Les barbiers avaient ajouté à leurs multiples activités médicales ou dentaires la pratique de la castration avec des instruments plus que primitifs et dans des conditions d'hygiène que l'on peut imaginer. Là, comme à l'hôpital, l'anesthésie demeurait le premier handicap: dans les meilleurs des cas, elle pouvait consister en l'absorption de breuvages contenant de l'opium afin de neutraliser au maximum les sensations de l'enfant. Plus souvent, on se contentait de compresser les carotides afin d'interrompre momentanément la circulation: l'enfant se trouvait alors dans un état proche du coma. On le plongeait ensuite dans un bain de lait pour amollir les parties génitales, ou dans un bain d'eau glacée qui jouait aussi un léger rôle anesthésiant et surtout empêchait de trop saigner.

La mortalité variait de 10 à $80 \%$ selon les opérateurs. L'opération n'avait jamais lieu avant sept ans et rarement après douze ans: il était essentiel qu'elle intervint avant le début de la fonction glandulaire des testicules. L'acte en lui-même devait être très rapide: on commençait par une incision à l'aine, par laquelle le chirurgien tirait le cordon et les testicules. L'ablation totale était alors effectuée par un couteau, tandis que les canaux étaient ligaturés. Cette opération différait beaucoup de celle des eunuques de harems à qui on enlevait tous les organes sexuels extérieurs.

La fin du XVIe siècle marqua le début de l'utilisation des castrats chanteurs dans la 
musique romaine. Encore peu connue dans l'Italie du XVIe siècle, hormis pour des raisons médicales précises, la pratique de la castration commença à se répandre dans les différents Etats de la Péninsule durant la première moitié du XVIIe siècle pour atteindre son point culminant au XVIIIe. Elle répondit à une mode, lancée en quelque sorte à la Chapelle Sixtine puis répandue à travers les cathédrales et les églises italiennes. Clément VIII fut immédiatement subjugué par les sopranistes et il autorisa la castration uniquement "ad honorem Dei". La vogue inouie des premiers grands sopranistes, sans parler de celle, plus tardive, d'un Farinelli ou d'un Gaffarelli, ne pouvait que pousser plus d'un père à sacrifier "pour son bien" l'un de ses rejetons. La démarche des parents était simple à comprendre: influencés par le triomphe des grands sopranistes et plus ou moins absous par l'autorité religieuse, ils voyaient dans la castration un moyen de soustraire leur fils à la condition modeste, voire à la misère qu'ils vivaient au quotidien, en lui assurant un avenir brillant, voué aux honneurs et à la fortune. Tous les castrats provenaient de famille très simples, impressionnées par l'avenir prometteur qu'on leur faisait miroiter. Un tel problème ne se posait pas aux familles aristocratiques, davantage soucieuses de voir leur fils transmettre la lignée ou accéder à de hautes charges ecclésiastiques.

\section{L'EVOLUTION DE LA VOIX DES CASTRATS}

Une fois castré l'enfant ne connaissait plus la mue; sa voix restait "haute", à mi-chemin entre celle de l'enfant et celle de la femme dont elle pouvait adopter l'une ou l'autre tessiture (soprano ou alto). La grande originalité du castrat tenait à la forme et à la position de son larynx. En effet, après la mue, le larynx de l'homme descend. Même la femme connaît une petite mue avec une très légère baisse du larynx sur son timbre. Le castrat ne connaissait pas cette descente du larynx, de sorte que ses cordes vocales restaient plus près des cavités de résonance. Ce larynx se rapprochait bien sur de celui de la femme tout en conservant la position, la forme et la plasticité de celui de l'enfant. Les atouts conjugués de ce larynx "hybride" trouvaient leur véritable épanouissement grâce à une prodigieuse musculature des cordes vocales, acquise patiemment au cours d'années de travail. De plus, la castration entrainait un développement important de la cage thoracique qui tendait à se bomber légèrement; elle devenait alors une appréciable caisse de résonance au service de cordes vocales de petite dimension, ce qui conférait à beaucoup une puissance que n'avaient pas les falsettistes. Différente de la voix d'homme par sa légèreté, ses flexibilités et ses aiguës, différente de la voix de femme par son éclat, sa limpidité et sa puissance, elle était également supérieure à celle de l'enfant par sa musculation d'adulte, sa technique et son expressivité. Le castrat modelait ainsi cette trinité hommefemme-enfant, pour en extraire une personnalité assurée et une voix souvent jugée sublime, divine et sensuelle [9].

\section{LE BOULEVERSEMENT PHYSIOLOGIQUE DES CASTRATS}

Certaines caractéristiques concernaient tous les castrats, telles que l'absence de pomme d'Adam ou une pilosité quasiment nulle, sauf peut-être dans la région pubienne puisque la sécrétion des glandes surrénales fournissaient encore quelques caractères masculins.

La plupart des castrats pouvaient connaître une relation sexuelle, la castration n'empêchant pas l'érection. Selon une expression de cette époque, les castrats présentaient une "impotentia generandi", mais non une 'impotentia cœundi"...Par rapport à leur "féminisation", les hormones féminines se trouvaient en effet suractivées par l'absence de testostérone, ce qui pouvait provoquer, par exemple, un plus ou moins grand développement des seins. De même le castrat acquérait une masse muscu- 
laire plus proche de celle de la femme, ainsi que des dépôts graisseux sur les hanches, les cuisses ou le cou [6].

Un autre caractéristique était la taille supérieure à la normale des castrats, phénomène plutôt gênant pour des personnages qui incarnaient parfois des rôles de femmes en dominant d'une tête leurs partenaires. Même si elle n'était pas généralisée, cette taille anormale s'expliquait par le fait que l'activité de l'hypohyse n'était pas contrebalancée par la testostérone et pouvait provoquer un effet renforcé de l'hormone de croissance. L'absence de testostérone faisait que les cartilages de conjugaison ne se soudaient pas après la puberté, et les os pouvaient encore s'allonger.

\section{LE XXéme SIECLE ET LES CASTRATS DU HAREM}

A l'aube du XXéme siècle, la castration se pratiquait encore en Europe sans cause d'intérêt chirurgical. Grâce aux travaux de l'Académie de Médecine sur les inconvénients de cette coutume barbare, le tsar a réprimé avec rigueur l'extension de la secte religieuse de "skoptzy", de ces fanatiques pour qui les organes de la génération étaient un objet d'abomination et qui pratiquaient la castration soit sur eux-mêmes, soit sur de tout jeunes enfants. Mais, c'était surtout chez les Orientaux que la castration était encore pratiquée [4]. Ils avaient conservé dans leurs mœurs cette pratique que l'Antiquité et le Moyen Age toléraient. L'Egypte était le pays où l'on faisait encore le commerce de castrats, et elle fournissait des eunuques au sérail du sultan Abdul-Hamid à Constantinople ainsi qu' à la cour du shah en Perse, au palais impérial de Pékin en Chine, au palais de l'empereur de Corée à Séoul, et en général aux harems des riches musulmans. Les opérateurs appartenaient à la secte religieuse des moines coptes. Ibn al Abbas, contemporain d'Abulcasis, décrivit en détails la technique de castration pour faire des eunuques, procédé qui est resté en usage jusqu'au début du XXème siècle. Ils achetaient à des marchands d'esclaves de jeunes nègres d'Abyssinie ou du Soudan âgés de sept à huit ans et les revendaient castrés [2].

L'opération, qu'ils pratiquaient sur ces malheureux était des plus primitives et des plus dangereuses. Sans aucun souci des principes de la chirurgie, ils saisissaient les parties génitales et les tranchaient en rasant le pubis, il ne devait rien rester des parties génitales. L'opéré était de suite placé dans un trou creusé dans le sable et restait ainsi enfoui jusqu'à la ceinture un temps suffisant pour que l'hémorragie soit arrêtée. Les deux tiers de ces malheureux périssaient ainsi victimes de ce honteux trafic. L'opéré s'il avait la chance de survivre à sa mutilation était conduit quelques mois plus tard au harem: là il passait sa vie au milieu des femmes qu'il serait chargé de surveiller à un âge plus avancé. Il se développait dans les profondeurs secrètes du palais et lorsqu'il avait atteint sa douzième année, il était encore impossible de le distinguer de ses autres camarades. L'âge de la puberté arrivait pour lui sans apporter aucun changement à sa physionomie d'enfant. Les eunuques, en dépit de leur mutilation, conservaient encore certaines sensations. Leurs fonctions consistaient à servir les femmes et les princes. Ils pouvaient entrer partout. Ils étaient au nombre d'environ 600 pour servir 300 femmes $\mathrm{du}$ harem de Constantinople. Autrefois, il y avait des eunuques blancs venus de Circassie. Le grand chef des eunuques était supérieur au maréchal. Enfin un code pénal spécial leur était affecté [11].

\section{REFERENCES}

1. Barbier P.: Histoire des Castrats. Edit. Grasset et Fasquelle, Paris, 1989,: 14-36.

2. Brtschal J., BRODNY M.L.: A history of Urology in Egypt. Ed.Riverside, New York, 1956,: 22-24.

3. DeRges V.F.: The keepers of the bed: Castration and religion. J.A.M.A., 1970, 212: 97-101.

4. Godard E.: Observations médicales et scientifiques. Egypte et Palestine, Ed. J.B .Baillière, Paris, 1867 ,: 11-17. 
5. Horton CH. E.: Plastic and Reconstructive Surgery of the Genital Area. Little, Brown and Company, Boston, 1973,: 8-15.

6. Minime L.: La médecine anecdotique, historique, littéraire. Ed. J.Rousset, Paris,1906,: 47-48.

7. ' PIRsche J.: De l'influence de la castration sur le développement du squelette. Ed. Storck \& Cic, lyon, 1902,: 6-14.

8. Pittard E.: La castration chez l'homme. Ed. Masson, Paris, 1934,: 17-22.

9. SPENCER R.F.: The cultural aspects of eunuchism. Ciba Symp.,1946, 8: 406-40g.

10. Verbeek Yv.: La sexualité dans la magie. Ed. Versoix et Idégraf, Genève, 1978,: 22-35.

11. ZeRvos SK.: La transplantation des testicules. Ed. Iatriki Proodos, Grèce, 1909,: 22-42.

\section{SUMMARY}

\section{Emasculation during the ages}

Georges Androutsos et Spyros Marketos

The first castration ever to be recorded,was indisputably the one of Osiris, performed by his own brother, Seth. Among the Greek Gods, Cronos mutilated his father-Uranus and threw the trophy in the sea. Among the Gods of Asia Minor, Attis, lover of Cybele, decided to commit self-emasculation, so as not to betray his divine mistress. Cybele intervened and resuscitated her lover,exalting him to divinity. His worship though,was blemished by a barbarous and bloody practice, that of the voluntary emasculation of the priests of Cybele. The Egyptians limited the sacrifice to the removal of the prepuce. The Hebrews found emasculation abhorrent, as did the early Christians. In Russia, at the beginning of the 18th century, a religious sect calling themselves 'Skoptzys' existed, who practised emasculation. This practice was abolished at the end of the 19th century, after the intense reaction of the tsar. At the dawn of the 20th century,emasculation is still practiced in the Orient, where eunuchs are required,as guardians for the harems. Egypt,was still the country where the commerce of eunuchs flourished and it provided eunuchs for the palace of the Sultan Abdulamid and other monarchs and rich muslims. The performers of such horrible mutilations were members of the religious sect of the Copts. During the Middle Ages the barbers realised that following castration,children developed and retained indefinitely a superb soprano voice. They started then looking for children with beautiful voices that they could castrate. For two centuries,between 1600 and 1800 , all the great opera singers,together with the musicians of the Catholic Church, were eunuchs. The most celebrated among those was Farinel1i (1705-1782). After the Napoleonic wars and the Decree-of 1902 of the Pope Leon XIII this barbaric custom was finally abolished.

Keywords : castration, punishment, opera, soprano, church, harem. 